\title{
Numerical Solution of Optimal Control Problems Governed by the Compressible Navier-Stokes Equations
}

\author{
S. Scott Collis, Kaveh Ghayour, Matthias Heinkenschloss, \\ Michael Ulbrich, and Stefan Ulbrich
}

\begin{abstract}
Theoretical and practical issues arising in optimal boundary control of the unsteady two-dimensional compressible Navier-Stokes equations are discussed. Assuming a sufficiently smooth state, formal adjoint and gradient equations are derived. For a vortex rebound model problem wall normal suction and blowing is used to minimize cost functionals of interest, here the kinetic energy at the final time.
\end{abstract}

\section{Introduction}

Recently, optimal control and optimal design problems governed by fluid flow models have received significant attention in the mathematical and in the engineering literature. See, e.g., the collections and reviews [7, 10,11]. The coupling of accurate computational fluid dynamics analyses with optimal control theory holds the promise for modifying a wide-range of fluid flows to achieve enhancement of desirable flow characteristics. Reduction of skin-friction drag, separation suppression, and increased lift to drag ratios for airfoils are examples of the types of optimization that such an approach enables. Moreover, advances in smart materials and microelectromechanical systems (MEMS) have increased the possibilities to actually implement controllers in physical systems. Optimal control problems have been studied mathematically and numerically for steady and unsteady incompressible Navier-Stokes flow. The references $[1,2,5,11,12]$ present a small sample of the work in this area. Compressible steady state Euler equations and Navier-Stokes equations have been used in the context of optimal design, see, e.g., [3, 14, 15, 16, 21].

In this paper we study the optimal control of two-dimensional unsteady compressible Navier-Stokes flows. To the best of the authors' knowledge, this is the first attempt to apply optimal control to problems governed by the unsteady compressible NavierStokes equations. Our research is motivated by the potential to develop novel and effective flow control strategies for inherently compressible phenomena including aeroacoustics and heat transfer by utilizing optimal control theory. Specifically, we plan to control the sound arising from Blade-Vortex Interaction (BVI) that can occur for rotorcraft in low speed, descending flight conditions, such as on approach to landing. When BVI occurs, 
tip vortices shed by a preceding blade interact with subsequent blades resulting in a high amplitude, impulsive noise that can dominate other rotorcraft noise sources. Reduction of the noise generated by this mechanism can alleviate restrictions on civil rotorcraft use near city centers and thereby enhance community acceptance. High frequency loading associated with this phenomenon also causes fatigue and hence reductions in BVI can have a direct impact on maintenance costs associated with blade failure in fatigue mode.

We use adjoint based gradient methods to solve the discretized optimal control problem. A critical issue in the numerical solution of optimal control problems is the accuracy of adjoint and gradient information. For successful optimization of the discretized problem, it is indispensable that the gradient approximation is sufficiently close to the derivative of the discretized objective function. To ensure that the solution of the discretized optimization problem approximates the infinite dimensional optimal control, it is also important that adjoint and gradient approximations used for the discretized problem converge towards their infinite dimensional counterparts as the discretization is refined. This requires a comprehensive view of the problem that integrates well posedness of the infinite dimensional problem, existence of adjoint equations and gradient equations, and properties of the discretization. Unfortunately, the mathematical foundation for optimal control problems governed by the unsteady compressible Navier-Stokes equations is not sufficiently developed to allow a rigorous and comprehensive study of gradient and adjoint accuracy in the previous sense. Even mathematical existence theories for the unsteady compressible Navier-Stokes equations are less developed than for the incompressible case.

In this paper, we discuss some of the theoretical issues arising in the formulation and solution of optimal boundary control problems governed by the compressible NavierStokes equations. Assuming a sufficiently smooth state, we derive formal adjoint and gradient equations. Finally, we present optimal control results for a vortex rebound test problem.

\section{Problem Formulation}

Let $\Omega=\left\{\mathrm{x} \in \mathbb{R}^{2}: x_{2}>0\right\}$ denote the spatial domain occupied by the fluid and let $\Gamma$ denote its spatial boundary. By

$$
\mathbf{u}=\left(\rho, v_{1}, v_{2}, T\right)^{T}
$$

we denote the primitive flow variables, where $\rho(t, \mathbf{x})$ is the density, $v_{i}(t, \mathbf{x})$ denotes the velocity in $x_{i}$-direction, $i=1,2, \mathbf{v}=\left(v_{1}, v_{2}\right)^{T}$, and $T(t, \mathbf{x})$ denotes the temperature. The pressure $p$ and the total energy per unit mass $E$ are given by

$$
p=\frac{\rho T}{\gamma \mathrm{M}^{2}}, \quad E=\frac{T}{\gamma(\gamma-1) \mathrm{M}^{2}}+\frac{1}{2} \mathbf{v}^{T} \mathbf{v},
$$

respectively, where $\gamma$ is the ratio of specific heats and $M$ is the reference Mach number. We write the conserved variables as functions of the primitive variables,

$$
\mathbf{q}(\mathbf{u})=\left(\rho, \rho v_{1}, \rho v_{2}, \rho E\right)^{T}
$$


and we define the inviscid flux terms

$$
\mathbf{F}^{1}(\mathbf{u})=\left(\begin{array}{c}
\rho v_{1} \\
\rho v_{1}^{2}+p \\
\rho v_{2} v_{1} \\
(\rho E+p) v_{1}
\end{array}\right), \quad \mathbf{F}^{2}(\mathbf{u})=\left(\begin{array}{c}
\rho v_{2} \\
\rho v_{1} v_{2} \\
\rho v_{2}^{2}+p \\
(\rho E+p) v_{2}
\end{array}\right),
$$

and the viscous flux terms

$$
\mathbf{G}^{i}(\mathbf{u}, \nabla \mathbf{u})=\frac{1}{\operatorname{Re}}\left(\begin{array}{c}
0 \\
\tau_{1 i} \\
\tau_{2 i} \\
\tau_{1 i} v_{1}+\tau_{2 i} v_{2}+\frac{\kappa}{\operatorname{PrM}^{2}(\gamma-1)} T_{x_{i}}
\end{array}\right)
$$

$i=1,2$, where $\tau_{i j}$ are the elements of the stress tensor $\tau=\mu\left(\nabla \mathbf{v}+\nabla \mathbf{v}^{T}\right)+\lambda(\nabla \cdot \mathbf{v}) I$. Here $\mu, \lambda$ are first and second coefficients of viscosity, $\kappa$ is the thermal conductivity, $\operatorname{Pr}$ is the reference Prandtl number, and Re is the reference Reynolds number. For the demonstration problems presented here, constant Prandtl number and fluid properties (viscosities and thermal conductivity) are assumed along with Stokes hypothesis for the second coefficient of viscosity, $\lambda=-2 \mu / 3$. Variable fluid properties can be easily accommodated and these effects will be included in future studies.

The two-dimensional compressible Navier-Stokes equations for the time interval $\left[t_{0}, t_{f}\right]$ can now be written as

$$
\begin{aligned}
\mathbf{q}(\mathbf{u})_{t}+\sum_{i=1}^{2}\left(\mathbf{F}^{i}(\mathbf{u})_{x_{i}}-\mathbf{G}^{i}(\mathbf{u}, \nabla \mathbf{u})_{x_{i}}\right) & =\mathbf{0} & & \text { in }\left(t_{0}, t_{f}\right) \times \Omega, \\
\mathbf{B}(\mathbf{u}, \nabla \mathbf{u}, \mathbf{g}) & =\mathbf{0} & & \text { on }\left(t_{0}, t_{f}\right) \times \Gamma, \\
\mathbf{u}\left(t_{0}, \mathbf{x}\right) & =\mathbf{u}_{0}(\mathbf{x}) & & \text { in } \Omega .
\end{aligned}
$$

The function $\mathbf{g}$ in the boundary conditions (4) acts as the control, which is taken to be suction and blowing in the wall normal direction on $\Gamma_{c} \subset \Gamma$. This is modeled by

$$
\mathbf{v}=\mathbf{b}+\mathbf{g} \quad \text { on } \Gamma_{c},
$$

where $\mathbf{b}$ is a given boundary velocity that satisfies the compatibility condition $\mathbf{v}\left(t_{0}, \mathbf{x}\right)=$ $\mathbf{b}\left(t_{0}, \mathbf{x}\right)$ for $\mathbf{x} \in \Gamma$. Since $\Gamma_{c} \subset\left\{\mathbf{x}: x_{2}=0\right\}$, we have $\mathbf{g}=\left(0, g_{2}\right)^{T}$.

To the best of our knowledge, the question of existence and uniqueness of global solutions for the full compressible Navier-Stokes equations (3)-(5) in 2D and 3D is still open for large initial data. The existence and uniqueness of global solutions with $(\rho-$ $\left.\rho_{0}, \mathbf{v}, T-T_{0}\right) \in C\left(t_{0}, t_{f} ; H^{3}\right) \cap C^{1}\left(t_{0}, t_{f} ; H^{1}\right)$ for the initial value problem (3), (5) is shown in [18] if the initial data $\mathbf{u}_{0}$ are close in $H^{3}$ to a constant state $\left(\rho_{0}, 0,0, T_{0}\right)^{T}$, $\rho_{0}, T_{0}>0$. Local existence in time can be shown also for large data. An analogous result for the initial-boundary value problem (3)-(5) on the half space or on the exterior of any bounded region with smooth boundary is shown in [19] for the boundary conditions $\left.\mathbf{v}\right|_{\left(t_{0}, t_{f}\right) \times \Gamma}=0$ and either $\left.T\right|_{\left(t_{0}, t_{f}\right) \times \Gamma}=T_{0}$ or $\frac{\partial T}{\partial \mathbf{n}}=0$, where $\mathbf{n}$ denotes the outward unit normal. Similar results can be found in the review article [22]. The global existence of weak solutions for the initial value problem is shown in [13], if $\rho\left(t_{0}, \cdot\right)-\rho_{0}$ is small in $L^{2} \cap L^{\infty}, \mathbf{v}\left(t_{0}, \cdot\right)$ is small in $L^{2} \cap L^{4}$, and $T\left(t_{0}, \cdot\right)-T_{0}$ is small in $L^{2}$ with constants 
$\rho_{0}, T_{0}>0$. It is shown that $\mathbf{v}, T$ are Hölder-continuous in space and time for $t>t_{0}$, $\mathbf{v}(t, \cdot), T(t, \cdot)-T_{0} \in H^{1}$, but merely $\rho(t, \cdot)-\rho_{0} \in L^{2} \cap L^{\infty}, \rho-\rho_{0} \in C\left(t_{0}, t_{f} ; H^{-1}\right)$. The question of uniqueness ist left open. Other results, mostly for the barotropic case in which pressure depends only on $\rho$ and which decouples the energy equation from the remaining ones, can be found in [17, 22].

The optimal control problem treated in this paper is the minimization of kinetic energy in $\Omega_{0} \subset \Omega$ at final time, more precisely,

$$
\begin{aligned}
\min _{\mathbf{g} \in \mathcal{G}} \widehat{J}(\mathbf{g}) \stackrel{\text { def }}{=} & \frac{1}{2} \int_{\Omega_{0}} \rho\left(t_{f}, \mathbf{x}\right)\left\|\mathbf{v}\left(t_{f}, \mathbf{x}\right)\right\|_{2}^{2} d \mathbf{x} \\
& +\int_{t_{0}}^{t_{f}} \int_{\Gamma_{c}}\left(\frac{\alpha_{1}}{2}\left\|\mathbf{g}_{t}\right\|_{2}^{2}+\frac{\alpha_{2}}{2}\|\nabla \mathbf{g}\|_{2}^{2}+\frac{\alpha_{3}}{2}\|\mathbf{g}\|_{2}^{2}\right) d \mathbf{x} d t
\end{aligned}
$$

where $\alpha_{1}, \alpha_{2}, \alpha_{3}>0$ and where the control space is chosen to be

$$
\mathcal{G}=\left\{\mathbf{g}: \mathbf{g} \in L^{2}\left(t_{0}, t_{f} ; H_{0}^{1}\left(\Gamma_{c}\right)\right), \mathbf{g}_{t} \in L^{2}\left(t_{0}, t_{f} ; L^{2}\left(\Gamma_{c}\right)\right), \mathbf{g}\left(t_{0}, \mathbf{x}\right)=0 \text { in } \Gamma_{c}\right\} .
$$

Here $\nabla \mathbf{g}$ is the gradient of $\mathbf{g}$ on the boundary, in our case $\nabla \mathbf{g}=\left(0,\left(g_{2}\right)_{x_{1}}\right)^{T}$. The second part of $\widehat{J}$ is a regularization term which, together with $\mathcal{G}$, must be chosen so that (7) is well-posed. In particular the regularity requirements on the control must be compatible with the regularity of the trace of $\mathbf{v}$ on $\Gamma_{c}$. For the incompressible Navier-Stokes equations such trace regularity estimates have been provided recently in $[8,9]$ and our choice of the control space and of the regularization term follows [12]. There is no theoretical justification yet that this choice is suitable for the compressible case. Significantly stronger regularity requirements on the controls seem necessary in connection with the theory in $[18,19]$. Our regularity requirements are closer aligned with what one would expect from the theory in [13]. Application of general existence results such as those in [8] to (7) are not yet known. However, our numerical results indicate that (7) is well-posed for the flows we have considered. A relaxation of the regularity requirements, i.e., setting $\alpha_{1}=0$ or even $\alpha_{1}=\alpha_{2}=0$ leads to highly oscillatory controls. More detailed grid convergence studies are under way. We remark that the choice of the control space and regularization term does not affect the adjoint equations computed in Sections 3.2, 3.3, it only effects how the gradient is computed given the adjoint (see (8)).

\section{Adjoint Equation}

\subsection{Adjoint Equation and Gradient for an Abstract Problem}

We first consider the gradient computation for an abstract functional whose evaluation involves implicitly defined functions. Let $\mathcal{G}$ be a Hilbert space with inner product $\langle\cdot, \cdot\rangle_{\mathcal{G}}$ and let $\mathcal{U}, \mathcal{C}$ be Banach spaces. We consider an equation $\mathbf{C}(\mathbf{u}, \mathbf{g})=\mathbf{0}$, where $\mathbf{C}: \mathcal{U} \times \mathcal{G} \rightarrow$ $\mathcal{C}$. Suppose that for every $\mathbf{g} \in \mathcal{G}$ the equation $\mathbf{C}(\mathbf{u}, \mathbf{g})=0$ has a unique solution $\mathbf{u}(\mathbf{g})$. We consider the abstract problem

$$
\min _{\mathbf{g} \in \mathcal{G}} \widehat{J}(\mathbf{g})=J(\mathbf{u}(\mathbf{g}), \mathbf{g})
$$


We assume the existence of neighborhoods $\bar{G}, \bar{U}$ of $\overline{\mathbf{g}}$ and $\overline{\mathbf{u}}=\mathbf{u}(\overline{\mathbf{g}})$, respectively, such that $\mathbf{C}$ is Fréchet-differentiable. Further, we assume that $\mathbf{u}(\mathrm{g})$ is differentiable on $\bar{G}$. This holds, e.g., if $\mathbf{C}$ is continuously Fréchet-differentiable on $\bar{U} \times \bar{G}$ and the partial derivative $\mathbf{C}_{\mathbf{u}}(\overline{\mathbf{u}}, \overline{\mathbf{g}})$ is continuously invertible. However, in our context these latter requirements seem to be too restrictive, since the results in [18, Prop. 4.1] indicate that the solution of the linearized state equation is less regular than the state $\overline{\mathbf{u}}$ about which the linearization is done. Now suppose that $J$ is Fréchet-differentiable on $\bar{U} \times \bar{G}$. Then the Fréchet-derivative $\widehat{J}_{\mathbf{g}}(\overline{\mathbf{g}}) \in \mathcal{G}^{*}$ and the gradient $\nabla \widehat{J}(\mathbf{g}) \in \mathcal{G}$ of $\widehat{J}$ can be computed from $\widehat{J}_{\mathbf{g}}(\overline{\mathbf{g}})=J_{\mathbf{u}}(\overline{\mathbf{u}}, \overline{\mathbf{g}}) \circ$ $\mathbf{u}_{\mathbf{g}}(\overline{\mathbf{g}})+J_{\mathbf{g}}(\overline{\mathbf{u}}, \overline{\mathbf{g}})$ and $\left\langle\widehat{J}_{\mathbf{g}}(\overline{\mathbf{g}}), \mathbf{g}^{\prime}\right\rangle_{\mathcal{G}^{*} \times \mathcal{G}}=\left\langle\nabla \widehat{J}(\overline{\mathbf{g}}), \mathbf{g}^{\prime}\right\rangle_{\mathcal{G}}$ for all $\mathbf{g}^{\prime} \in \mathcal{G}$, respectively, where $\mathcal{G}^{*}$ denotes the topological dual of $\mathcal{G}$ and $\langle\cdot, \cdot\rangle_{\mathcal{G}^{*} \times \mathcal{G}}$ denotes the duality pairing between $\mathcal{G}^{*}$ and $\mathcal{G}$.

It can be shown that the gradient $\nabla \widehat{J}(\overline{\mathbf{g}})$ can be computed from

$$
\left\langle\nabla \widehat{J}(\overline{\mathbf{g}}), \mathbf{g}^{\prime}\right\rangle_{\mathcal{G}}=\left\langle\mathbf{C}_{\mathbf{g}}(\overline{\mathbf{u}}, \overline{\mathbf{g}})^{*} \bar{\lambda}, \mathbf{g}^{\prime}\right\rangle_{\mathcal{G}^{*} \times \mathcal{G}}+\left\langle J_{\mathbf{g}}(\overline{\mathbf{u}}, \overline{\mathbf{g}}), \mathbf{g}^{\prime}\right\rangle_{\mathcal{G}^{*} \times \mathcal{G}}
$$

for all $\mathbf{g}^{\prime} \in \mathcal{G}$, if there exists an adjoint state $\bar{\lambda} \in \mathcal{C}^{*}$ satisfying the adjoint equation $\mathbf{C}_{\mathbf{u}}(\overline{\mathbf{u}}, \overline{\mathbf{g}})^{*} \overline{\boldsymbol{\lambda}}=-J_{\mathbf{u}}(\overline{\mathbf{u}}, \overline{\mathbf{g}})$ in $\mathcal{U}^{*}$, i.e., if $\bar{\lambda}$ satisfies

$$
\left\langle\mathbf{C}_{\mathbf{u}}(\overline{\mathbf{u}}, \overline{\mathbf{g}})^{*} \bar{\lambda}, \mathbf{u}^{\prime}\right\rangle_{\mathcal{U}^{*} \times \mathcal{U}}=\left\langle-J_{\mathbf{u}}(\overline{\mathbf{u}}, \overline{\mathbf{g}}), \mathbf{u}^{\prime}\right\rangle_{\mathcal{U}^{*} \times \mathcal{U}}
$$

for all $\mathbf{u}^{\prime} \in \mathcal{U}$. The existence of $\bar{\lambda}$ is ensured if $\mathbf{C}_{\mathbf{u}}(\overline{\mathbf{u}}, \overline{\mathbf{g}})^{*}$ is onto. Note, however, that it is sufficient that the adjoint equation is solvable for the particular right hand side $-J_{\mathbf{u}}(\overline{\mathbf{u}}, \overline{\mathbf{g}})$.

\subsection{Adjoint Equation for the Compressible Navier-Stokes Equations}

We carry out the formal derivation of the adjoint equation for the general situation that $\Omega$ is a domain with $C^{2}$-boundary $\Gamma$. According to (3)-(5) we define

$$
\mathbf{C}(\mathbf{u}, \mathbf{g})=\left(\begin{array}{c}
\mathbf{q}(\mathbf{u})_{t}+\sum_{i=1}^{2}\left(\mathbf{F}^{i}(\mathbf{u})-\mathbf{G}^{i}(\mathbf{u}, \nabla \mathbf{u})\right)_{x_{i}} \\
\mathbf{B}(\mathbf{u}, \nabla \mathbf{u}, \mathbf{g}) \\
\mathbf{u}-\mathbf{u}_{0}
\end{array}\right) .
$$

To write the linearization of the Navier-Stokes equation it is useful to define $\mathbf{M}=$ $\mathbf{q}_{\mathbf{u}}(\mathbf{u}), \mathbf{A}^{i}=\mathbf{F}_{\mathbf{u}}^{i}(\mathbf{u}), i=1,2$, and to write

$$
\mathbf{G}^{i}(\mathbf{u}, \nabla \mathbf{u})=\mathbf{K}_{1}^{i}(\mathbf{u}) \mathbf{u}_{x_{1}}+\mathbf{K}_{2}^{i}(\mathbf{u}) \mathbf{u}_{x_{2}}, \quad i=1,2,
$$

where

$$
\begin{aligned}
\mathbf{K}_{1}^{1}(\mathbf{u}) & =\frac{1}{\operatorname{Re}}\left(\begin{array}{cccc}
0 & 0 & 0 & 0 \\
0 & \widetilde{\mu} & 0 & 0 \\
0 & 0 & \mu & 0 \\
0 & \widetilde{\mu} v_{1} & \mu v_{2} & \frac{\kappa}{\operatorname{PrM}^{2}(\gamma-1)}
\end{array}\right), \quad \mathbf{K}_{2}^{1}(\mathbf{u})=\frac{1}{\operatorname{Re}}\left(\begin{array}{ccccc}
0 & 0 & 0 & 0 \\
0 & 0 & \lambda & 0 \\
0 & \mu & 0 & 0 \\
0 & \mu v_{2} & \lambda v_{1} & 0
\end{array}\right), \\
\mathbf{K}_{1}^{2}(\mathbf{u}) & =\frac{1}{\operatorname{Re}}\left(\begin{array}{ccccc}
0 & 0 & 0 & 0 \\
0 & 0 & \mu & 0 \\
0 & \lambda & 0 & 0 \\
0 & \lambda v_{2} & \mu v_{1} & 0
\end{array}\right), \quad \mathbf{K}_{2}^{2}(\mathbf{u})=\frac{1}{\operatorname{Re}}\left(\begin{array}{cccc}
0 & 0 & 0 & 0 \\
0 & \mu & 0 & 0 \\
0 & 0 & \widetilde{\mu} & 0 \\
0 & \mu v_{1} & \tilde{\mu} v_{2} & \frac{\kappa}{\operatorname{PrM}^{2}(\gamma-1)}
\end{array}\right)
\end{aligned}
$$


with $\widetilde{\mu}=2 \mu+\lambda$. From the representation (11) we obtain $\mathbf{G}_{\mathbf{u}_{x_{j}}}^{i}(\mathbf{u}, \nabla \mathbf{u})=\mathbf{K}_{j}^{i}(\mathbf{u})$, $i, j=1,2$, and the definition (2) of the viscous terms implies

$$
\mathbf{G}_{\mathbf{u}}^{i}(\mathbf{u}, \nabla \mathbf{u})=\mathbf{D}^{i}(\nabla \mathbf{u})=\frac{1}{\operatorname{Re}}\left(\begin{array}{cccc}
0 & 0 & 0 & 0 \\
0 & 0 & 0 & 0 \\
0 & 0 & 0 & 0 \\
0 & \tau_{1 i} & \tau_{2 i} & 0
\end{array}\right), \quad i=1,2
$$

In the following we simply write $\mathbf{K}_{j}^{i}$ instead of $\mathbf{K}_{j}^{i}(\mathbf{u}), i, j=1,2$, and $\mathbf{D}^{i}$ instead of $\mathbf{D}^{i}(\nabla \mathbf{u}), i=1,2$. With this notation, the linearized state equation can be written as

$$
\mathbf{C}_{\mathbf{u}}(\mathbf{u}, \mathbf{g}) \mathbf{u}^{\prime}=\left(\begin{array}{c}
\left(\mathbf{M} \mathbf{u}^{\prime}\right)_{t}+\sum_{i}\left(\mathbf{A}^{i} \mathbf{u}^{\prime}-\mathbf{D}^{i} \mathbf{u}^{\prime}-\sum_{j} \mathbf{K}_{j}^{i} \mathbf{u}_{x_{j}}^{\prime}\right)_{x_{i}} \\
\mathbf{B}_{\mathbf{u}}(\mathbf{u}, \nabla \mathbf{u}, \mathbf{g}) \mathbf{u}^{\prime}+\sum_{j} \mathbf{B}_{\mathbf{u}_{x_{j}}}(\mathbf{u}, \nabla \mathbf{u}, \mathbf{g}) \mathbf{u}_{x_{j}}^{\prime} \\
\mathbf{u}^{\prime}
\end{array}\right)
$$

The adjoint variables $\boldsymbol{\lambda}$ are partitioned according to the partition of $\mathbf{C}$ in (10) and are denoted by $\boldsymbol{\lambda}=\left(\boldsymbol{\lambda}^{d}, \boldsymbol{\lambda}^{b}, \boldsymbol{\lambda}^{0}\right)$.

We assume that

$$
\begin{aligned}
\left\langle D_{\mathbf{u}} J(\mathbf{u}, \mathbf{g}), \mathbf{u}^{\prime}\right\rangle_{\mathcal{U}^{*} \times \mathcal{U}}= & \int_{t_{0}}^{t_{f}} \int_{\Omega}\left(\mathbf{u}^{\prime}\right)^{T} \mathbf{r}+\int_{\Omega}\left(\left.\mathbf{u}^{\prime}\right|_{t=t_{f}}\right)^{T} \mathbf{r}_{t_{f}} \\
& +\int_{t_{0}}^{t_{f}} \int_{\Gamma}\left(\left(\mathbf{u}^{\prime}\right)^{T} \mathbf{r}_{\Gamma}+\left(\nabla \mathbf{u}^{\prime} \mathbf{n}\right)^{T} \mathbf{r}_{\Gamma, \mathbf{n}}+\left(\nabla \mathbf{u}^{\prime} \mathbf{s}\right)^{T} \mathbf{r}_{\Gamma, \mathbf{s}}\right),
\end{aligned}
$$

where $\mathbf{n}=\left(n_{1}, n_{2}\right)^{T}$ is the unit outward normal, $\mathbf{s}=\left(s_{1}, s_{2}\right)^{T}=\left(-n_{2}, n_{1}\right)^{T}$ is the unit tangential vector, and $\nabla \mathbf{u}^{\prime}$ is the Jacobian of $\mathbf{u}^{\prime}$. This is true for the objective function in (7), but also for many more general objective functions that involve distributed observations or observations of normal derivatives $\nabla \mathbf{u} \mathbf{n}$ or of tangential derivatives $\nabla \mathbf{u} \mathbf{s}$ of the state.

To derive the adjoint equations, we multiply $\mathbf{C}_{\mathbf{u}}(\mathbf{u}, \mathbf{g}) \mathbf{u}^{\prime}$ by $\boldsymbol{\lambda}$ and integrate the resulting terms over $\left(t_{0}, t_{f}\right) \times \Omega,\left(t_{0}, t_{f}\right) \times \Gamma$, and $\Omega$, respectively. Integration by parts 
leads to (9), which in this case is given by

$$
\begin{aligned}
& -\int_{t_{0}}^{t_{f}} \int_{\Omega}\left(\mathbf{u}^{\prime}\right)^{T} \mathbf{r}-\int_{\Omega}\left(\left.\mathbf{u}^{\prime}\right|_{t=t_{f}}\right)^{T} \mathbf{r}_{t_{f}} \\
& \quad-\int_{t_{0}}^{t_{f}} \int_{\Gamma}\left(\left(\mathbf{u}^{\prime}\right)^{T} \mathbf{r}_{\Gamma}+\left(\nabla \mathbf{u}^{\prime} \mathbf{n}\right)^{T} \mathbf{r}_{\Gamma, \mathbf{n}}+\left(\nabla \mathbf{u}^{\prime} \mathbf{s}\right)^{T} \mathbf{r}_{\Gamma, \mathbf{s}}\right) \\
& =\int_{t_{0}}^{t_{f}} \int_{\Omega}\left(\mathbf{u}^{\prime}\right)^{T}\left(-\mathbf{M}^{T} \boldsymbol{\lambda}_{t}^{d}-\sum_{i}\left(\left(\mathbf{A}^{i}-\mathbf{D}^{i}\right)^{T} \boldsymbol{\lambda}_{x_{i}}^{d}+\sum_{j}\left(\left(\mathbf{K}_{j}^{i}\right)^{T} \boldsymbol{\lambda}_{x_{i}}^{d}\right)_{x_{j}}\right)\right) \\
& \quad+\int_{t_{0}}^{t_{f}} \int_{\Gamma}\left(\mathbf{u}^{\prime}\right)^{T}\left(\sum_{i}\left(n_{i}\left(\mathbf{A}^{i}-\mathbf{D}^{i}\right)^{T} \boldsymbol{\lambda}^{d}+\sum_{j} n_{j}\left(\mathbf{K}_{j}^{i}\right)^{T} \boldsymbol{\lambda}_{x_{i}}^{d}\right)+\mathbf{B}_{\mathbf{u}}^{T} \boldsymbol{\lambda}^{b}\right) \\
& \quad+\int_{t_{0}}^{t_{f}} \int_{\Gamma} \sum_{j}\left(\mathbf{u}_{x_{j}}^{\prime}\right)^{T}\left(\mathbf{B}_{\mathbf{u}_{x_{j}}}^{T} \boldsymbol{\lambda}^{b}-\sum_{i} n_{i}\left(\mathbf{K}_{j}^{i}\right)^{T} \boldsymbol{\lambda}^{d}\right) \\
& \quad+\left.\int_{\Omega}\left(\mathbf{u}^{\prime}\right)^{T} \mathbf{M}^{T} \boldsymbol{\lambda}^{d}\right|_{t=t_{f}}+\left.\int_{\Omega}\left(\mathbf{u}^{\prime}\right)^{T}\left(\boldsymbol{\lambda}^{0}-\mathbf{M}^{T} \boldsymbol{\lambda}^{d}\right)\right|_{t=t_{0}} \quad \forall \mathbf{u}^{\prime} .
\end{aligned}
$$

If we choose test functions $\mathbf{u}^{\prime} \in C_{0}^{\infty}\left(\left(t_{0}, t_{f}\right) \times \Omega\right)$, then (14) implies

$$
\mathbf{M}^{T} \boldsymbol{\lambda}_{t}^{d}+\sum_{i}\left(\left(\mathbf{A}^{i}-\mathbf{D}^{i}\right)^{T} \boldsymbol{\lambda}_{x_{i}}^{d}+\sum_{j}\left(\left(\mathbf{K}_{j}^{i}\right)^{T} \boldsymbol{\lambda}_{x_{i}}^{d}\right)_{x_{j}}\right)=\mathbf{r}
$$

in $\left(t_{0}, t_{f}\right) \times \Omega$. If we choose test functions $\mathbf{u}^{\prime}$ such that $\mathbf{u}^{\prime}=0$ on $\left\{t_{0}\right\} \times \Omega, \mathbf{u}^{\prime}=0$ and $\nabla \mathbf{u}^{\prime}=0$ on $\left(t_{0}, t_{f}\right) \times \Gamma$, then (14) implies $\left.\left(\mathbf{M}^{T} \boldsymbol{\lambda}^{d}\right)\right|_{t=t_{f}}=-\mathbf{r}_{t_{f}}$ in $\Omega$ or, equivalently,

$$
\lambda^{d}=-\mathbf{M}^{-T} \mathbf{r}_{t_{f}} \quad \text { in }\left\{t_{f}\right\} \times \Omega .
$$

Similarly, if we choose test functions $\mathbf{u}^{\prime}$ such that $\mathbf{u}^{\prime}=0$ on $\left\{t_{f}\right\} \times \Omega, \mathbf{u}^{\prime}=0$ and $\nabla \mathbf{u}^{\prime}=0$ on $\left(t_{0}, t_{f}\right) \times \Gamma$, then (14) implies $\boldsymbol{\lambda}^{0}-\left.\left(\mathbf{M}^{T} \boldsymbol{\lambda}^{d}\right)\right|_{t=t_{0}}=0$ in $\Omega$. This means that $\boldsymbol{\lambda}^{0}$ is determined by $\left.\boldsymbol{\lambda}^{d}\right|_{t=t_{0}}$.

Next we choose test functions $\mathbf{u}^{\prime}$ such that $\mathbf{u}^{\prime}=0$ on $\left\{0, t_{f}\right\} \times \Omega$ and on $\left(t_{0}, t_{f}\right) \times \Gamma$. The tangential derivatives $\left(\nabla \mathbf{u}^{\prime}\right)$ s of these test functions is zero. Using $\nabla \mathbf{u}^{\prime}=\nabla \mathbf{u}^{\prime} \mathbf{n n}^{T}+$ $\nabla \mathbf{u}^{\prime} \mathbf{s s}^{T}=\nabla \mathbf{u}^{\prime} \mathbf{n} \mathbf{n}^{T}, \mathbf{u}_{x_{j}}^{\prime}=\nabla \mathbf{u}^{\prime} e_{j}=\nabla \mathbf{u}^{\prime} \mathbf{n} n_{j}$ and the previous identities in (14), we obtain

$$
\sum_{j} n_{j}\left(\mathbf{B}_{\mathbf{u}_{x_{j}}^{T}}^{T} \boldsymbol{\lambda}^{b}-\sum_{i} n_{i}\left(\mathbf{K}_{j}^{i}\right)^{T} \boldsymbol{\lambda}^{d}\right)=-\mathbf{r}_{\Gamma, n} \quad \text { on }\left(t_{0}, t_{f}\right) \times \Gamma .
$$


With (15)-(17) the identity (14) reduces to

$$
\begin{aligned}
-\int_{t_{0}}^{t_{f}} & \int_{\Gamma}\left(\left(\mathbf{u}^{\prime}\right)^{T} \mathbf{r}_{\Gamma}+\left(\nabla \mathbf{u}^{\prime} \mathbf{s}\right)^{T} \mathbf{r}_{\Gamma, \mathbf{s}}\right) \\
= & \int_{t_{0}}^{t_{f}} \int_{\Gamma}\left(\mathbf{u}^{\prime}\right)^{T}\left(\sum_{i}\left(n_{i}\left(\mathbf{A}^{i}-\mathbf{D}^{i}\right)^{T} \boldsymbol{\lambda}^{d}+\sum_{j} n_{j}\left(\mathbf{K}_{j}^{i}\right)^{T} \boldsymbol{\lambda}_{x_{i}}^{d}\right)+\mathbf{B}_{\mathbf{u}}^{T} \boldsymbol{\lambda}^{b}\right) \\
& +\int_{t_{0}}^{t_{f}} \int_{\Gamma}\left(\nabla \mathbf{u}^{\prime} \mathbf{s}\right)^{T} \sum_{j} s_{j}\left(\mathbf{B}_{\mathbf{u}_{x_{j}}}^{T} \boldsymbol{\lambda}^{b}-\sum_{i} n_{i}\left(\mathbf{K}_{j}^{i}\right)^{T} \boldsymbol{\lambda}^{d}\right) \quad \forall \mathbf{u}^{\prime} .
\end{aligned}
$$

One can employ integration by parts over $\Gamma$ on the integrals involving $\left(\nabla \mathbf{u}^{\prime} \mathbf{s}\right)$ to arrive at the more convenient identity

$$
\begin{aligned}
& \sum_{i}\left(n_{i}\left(\mathbf{A}^{i}-\mathbf{D}^{i}\right)^{T} \boldsymbol{\lambda}^{d}+\sum_{j} n_{j}\left(\mathbf{K}_{j}^{i}\right)^{T} \boldsymbol{\lambda}_{x_{i}}^{d}\right)+\mathbf{B}_{\mathbf{u}}^{T} \boldsymbol{\lambda}^{b} \\
& -\frac{\partial}{\partial \mathbf{s}}\left(\sum_{j} s_{j}\left(\mathbf{B}_{\mathbf{u}_{x_{j}}}^{T} \boldsymbol{\lambda}^{b}-\sum_{i} n_{i}\left(\mathbf{K}_{j}^{i}\right)^{T} \boldsymbol{\lambda}^{d}\right)+\mathbf{r}_{\Gamma, \mathbf{s}}\right)=-\mathbf{r}_{\Gamma} .
\end{aligned}
$$

\subsection{Adjoint Equation for the Boundary Control of Final-Time Kinetic Energy}

In our model problem (7) we assume adiabatic boundary conditions for the temperature on the bottom wall $\Gamma=\left\{\mathbf{x} \in \mathbb{R}^{2}: x_{2}=0\right\}$. The velocities on $\Gamma$ are prescribed and are equal to $\mathbf{b}$ on $\left(t_{0}, t_{f}\right) \times\left(\Gamma \backslash \Gamma_{c}\right)$ and they are equal to $\mathbf{b}+\mathbf{g}$ on $\left(t_{0}, t_{f}\right) \times \Gamma_{c}$. The boundary condition operator $\mathbf{B}$ in (4) is

$$
\mathbf{B}(\mathbf{u}, \nabla \mathbf{u}, \mathbf{g})=\left(\begin{array}{c}
\mathbf{v}-\mathbf{g}-\mathbf{b} \\
-T_{x_{2}}
\end{array}\right), \quad \mathbf{B}(\mathbf{u}, \nabla \mathbf{u}, \mathbf{g})=\left(\begin{array}{c}
\mathbf{v}-\mathbf{b} \\
-T_{x_{2}}
\end{array}\right)
$$

on $\left(t_{0}, t_{f}\right) \times \Gamma_{c}$ and on $\left(t_{0}, t_{f}\right) \times\left(\Gamma \backslash \Gamma_{c}\right)$, respectively. The partial Fréchet-derivative of the objective function $J$ in (7) is given by (13) with $\mathbf{r}=\mathbf{r}_{\Gamma, \mathbf{n}}=\mathbf{r}_{\Gamma, \mathbf{s}}=\mathbf{0}$ and

$$
\mathbf{r}_{t_{f}}(\mathbf{x})=\mathbf{1}_{\Omega_{0}}(\mathbf{x})\left(\frac{1}{2}\left\|\mathbf{v}\left(\mathbf{x}, t_{f}\right)\right\|_{2}^{2}, \rho\left(\mathbf{x}, t_{f}\right) \mathbf{v}\left(\mathbf{x}, t_{f}\right), 0\right)^{T} .
$$

Since $\Omega=\left\{\mathbf{x} \in \mathbb{R}^{2}: x_{2}>0\right\}, \mathbf{n}=(0,-1)^{T}$ on $\Gamma$, the boundary condition (17) reads

$$
\mathbf{B}_{\mathbf{u}_{x_{2}}}^{T} \boldsymbol{\lambda}^{b}+\left(\mathbf{K}_{2}^{2}\right)^{T} \boldsymbol{\lambda}^{d}=0, \quad \text { on }\left(t_{0}, t_{f}\right) \times \Gamma,
$$

which, using the definition of $\mathbf{B}$ and $\mathbf{K}_{2}^{2}$, is equivalent to

$$
\frac{1}{\operatorname{Re}}\left(\begin{array}{ccc}
\mu & 0 & \mu v_{1} \\
0 & 2 \mu+\lambda & (2 \mu+\lambda) v_{2} \\
0 & 0 & \frac{\kappa}{\operatorname{PrM}^{2}(\gamma-1)}
\end{array}\right)\left(\begin{array}{c}
\lambda_{2}^{d} \\
\lambda_{3}^{d} \\
\lambda_{4}^{d}
\end{array}\right)=\left(\begin{array}{c}
0 \\
0 \\
\lambda_{3}^{b}
\end{array}\right) \quad \text { on }\left(t_{0}, t_{f}\right) \times \Gamma .
$$

Hence, we obtain

$$
\lambda_{3}^{b}=\frac{\kappa}{\operatorname{RePrM}^{2}(\gamma-1)} \lambda_{4}^{d} \text { on }\left(t_{0}, t_{f}\right) \times \Gamma,
$$


and the boundary conditions

$$
\lambda_{2}^{d}=-v_{1} \lambda_{4}^{d}, \quad \lambda_{3}^{d}=-v_{2} \lambda_{4}^{d} \quad \text { on }\left(t_{0}, t_{f}\right) \times \Gamma .
$$

These boundary conditions imply $\left(\mathbf{K}_{1}^{2}\right)^{T} \boldsymbol{\lambda}^{d}=0$. Hence, the boundary condition (18) on $\left(t_{0}, t_{f}\right) \times \Gamma$ reduces to

$$
\left(\mathbf{A}^{2}-\mathbf{D}^{2}\right)^{T} \boldsymbol{\lambda}^{d}+\left(\mathbf{K}_{2}^{1}\right)^{T} \boldsymbol{\lambda}_{x_{1}}^{d}+\left(\mathbf{K}_{2}^{2}\right)^{T} \boldsymbol{\lambda}_{x_{2}}^{d}=\mathbf{B}_{\mathbf{u}}^{T} \boldsymbol{\lambda}^{b} .
$$

By definition, $\mathbf{A}^{2}=\mathbf{F}_{\mathbf{u}}^{2}(\mathbf{u})$, where $\mathbf{F}^{2}(\mathbf{u})$ is one of the inviscid fluxes in (1). Hence,

$$
\mathbf{A}^{2}=\left(\begin{array}{cccc}
v_{2} & 0 & \rho & 0 \\
v_{1} v_{2} & \rho v_{2} & \rho v_{1} & 0 \\
v_{2}^{2}+\frac{T}{\gamma \mathrm{M}^{2}} & 0 & 2 \rho v_{2} & \frac{\rho}{\gamma \mathrm{M}^{2}} \\
v_{2}\left(\frac{T}{(\gamma-1) \mathrm{M}^{2}}+\frac{1}{2} \mathbf{v}^{T} \mathbf{v}\right) & \rho v_{1} v_{2} & \rho\left(\frac{T}{(\gamma-1) \mathrm{M}^{2}}+\frac{1}{2} \mathbf{v}^{T} \mathbf{v}\right)+\rho v_{2}^{2} & \frac{\rho v_{2}}{(\gamma-1) \mathrm{M}^{2}}
\end{array}\right) .
$$

Moreover, using the definition of $\mathbf{K}_{2}^{1}$ and (21) we obtain

$$
\left(\mathbf{K}_{2}^{1}\right)^{T} \boldsymbol{\lambda}_{x_{1}}^{d}=\frac{1}{\operatorname{Re}}\left(\begin{array}{c}
0 \\
\mu\left(\lambda_{3}^{d}\right)_{x_{1}}+\mu v_{2}\left(\lambda_{4}^{d}\right)_{x_{1}} \\
\lambda\left(\lambda_{2}^{d}\right)_{x_{1}}+\lambda v_{1}\left(\lambda_{4}^{d}\right)_{x_{1}} \\
0
\end{array}\right)=\frac{1}{\operatorname{Re}}\left(\begin{array}{c}
0 \\
-\mu\left(v_{2}\right)_{x_{1}} \\
-\lambda\left(v_{1}\right)_{x_{1}} \\
0
\end{array}\right) \lambda_{4}^{d} .
$$

If we insert the previous two equations and (21) into (22) we arrive at the condition

$$
\begin{gathered}
\left(\begin{array}{cc}
v_{2} & v_{2}\left(\frac{T}{\gamma\left(\gamma-1 \mathrm{M}^{2}\right.}-\frac{1}{2} \mathbf{v}^{T} \mathbf{v}\right) \\
0 & -\frac{1}{\operatorname{Re}}\left(\mu\left(v_{2}\right)_{x_{1}}+\tau_{12}\right) \\
\rho & \rho\left(\frac{T}{(\gamma-1) \mathrm{M}^{2}}-\frac{1}{2} \mathbf{v}^{T} \mathbf{v}\right)-\frac{1}{\operatorname{Re}}\left(\lambda\left(v_{1}\right)_{x_{1}}+\tau_{22}\right)
\end{array}\right)\left(\begin{array}{c}
\lambda_{1}^{d} \\
\lambda_{4}^{d}
\end{array}\right) \\
\begin{array}{c}
\rho v_{2} \\
0
\end{array} \\
+\frac{1}{\operatorname{Re}}\left(\begin{array}{ccc}
0 & 0 & 0 \\
\mu & 0 & \mu v_{1} \\
0 & 2 \mu+\lambda & (2 \mu+\lambda) v_{2} \\
0 & 0 & \frac{\kappa}{(\gamma-1) \mathrm{M}^{2} \operatorname{Pr}}
\end{array}\right)\left(\begin{array}{c}
\lambda_{2}^{d} \\
\lambda_{3}^{d} \\
\lambda_{4}^{d}
\end{array}\right)_{x_{2}}=\left(\begin{array}{c}
0 \\
\lambda_{1}^{b} \\
\lambda_{2}^{b} \\
0
\end{array}\right)
\end{gathered}
$$

on $\left(t_{0}, t_{f}\right) \times \Gamma$. Equation (23) yields the boundary conditions

$$
\begin{aligned}
v_{2} \lambda_{1}^{d} & =v_{2}\left(-\frac{T}{\gamma(\gamma-1) \mathrm{M}^{2}}+\frac{1}{2} \mathbf{v}^{T} \mathbf{v}\right) \lambda_{4}^{d}, \\
\rho v_{2} \lambda_{4}^{d} & =-\frac{\kappa}{(\gamma-1) \mathrm{M}^{2} \operatorname{PrRe}}\left(\lambda_{4}^{d}\right)_{x_{2}}
\end{aligned}
$$

on $\left(t_{0}, t_{f}\right) \times \Gamma$. Thus, the adjoint boundary conditions for $\boldsymbol{\lambda}^{d}$ are given by (21), (24). If desired, the adjoint variables $\lambda^{b}$ for the boundary data can be computed from $\lambda^{d}$ using (20) and the second and third equation in (23).

We remark, that the general formulation of the adjoint equations (15)-(18) is also useful, when non-reflecting boundary conditions are introduced on the boundary $\partial \Omega_{c} \backslash \Gamma$ of the computational domain $\Omega_{c} \subset \Omega$. 


\subsection{Gradient Computation}

Given the adjoint $\lambda$, the gradient can be computed from (8). As in $[9,12]$ this leads to an elliptic problem on the time-space boundary $\left(t_{0}, t_{f}\right) \times \Gamma$ for $\nabla \widehat{J}$. Due to space restrictions the details are omitted here. For the numerical solution of the optimal control problem the solution of this PDE can be avoided with a reformulation of the optimal control problem and working with a different, yet appropriate inner product. This is described in [4] for the semi-discrete case.

\section{Results}

We present results for a model problem consisting of two counter-rotating viscous vortices above an infinite wall which, due to the self-induced velocity field, propagate downward and interact with the wall. Our non-dimensionalization is based on initial vortex core radius and the maximum azimuthal velocities at the edge of the viscous cores. For the computations reported here, the Mach, Reynolds, and Prandtl numbers are $\mathrm{M}=$ $0.5, \operatorname{Re}=25, \operatorname{Pr}=1$, respectively. Our computational domain is $[-15,15] \times[0,15]$ in non-dimensional units. The compressible Navier-Stokes equations are discretized in space using fourth-order accurate central differences on a $128 \times 128$ uniform grid. Time integration is performed using the classical fourth-order Runge Kutta method with a fixed time step $\Delta t=0.05$. To compute the initial conditions, two compressible Oseen vortices [6], located at $( \pm 2,7.5)$, are superimposed at time $t=0$. From this superposed field, which is not a solution to the equations, we advance 100 time steps until time $t_{0}=5$, and take the resulting flow field at time $t_{0}$ as the initial condition to our problem. The side and top boundaries are assumed to be located far enough from the main flow region to justify the imposition of a characteristics based inviscid far-field boundary condition. For details on the model problem and its discretization see [4].

We control the flow in the time window $t_{0}=5, t_{f}=40$. Our control $\mathbf{g}$ is the wall normal velocity which for our geometry is given by $\mathbf{g}=\left(0, g_{2}\right)$. The following control plots show $g_{2}$. Positive $g_{2}$ represents injection (blowing) of fluid into the domain while negative $g_{2}$ corresponds to suction of fluid out of the domain.

Our numerical results are produced using a nonlinear conjugate gradient (NCG) algorithm [20] for the solution of the discretized problem. The inner products used in the NCG method are discretizations of the $\mathcal{G}$ inner product (see [4]) to minimize the meshdependent behavior of the cg method and to avoid artificial ill-conditioning due to discretization. All computations are performed in parallel on an SGI Origin 2000. Using four processors, one optimization run takes about 10hours.

We performed three runs, two include a regularization of the time derivative of the control, the third does not. The coefficients $\alpha_{j}$ in the regularization term, the value of the objective functional in (7) at the initial iterate, i.e., for zero control $\left(J_{0}\right)$, at the final control iterate $\left(J_{\text {final }}\right)$ and the terminal kinetic energy (the first integral in (7)) at the final control iterate $\left(\mathrm{TKE}_{\text {final }}\right)$ are shown in Table 1 . We see that because of the large regularization parameter $\alpha_{1}$, the terminal kinetic energy reduction in run I is less than that for run III. 
FIGURE 1. Optimal wall-normal velocity distributions

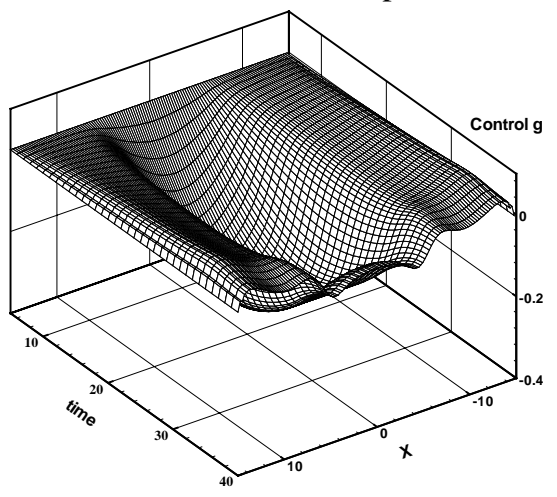

Optimal control run I

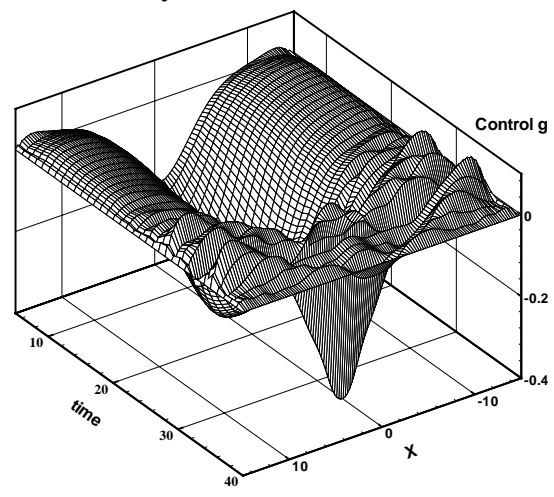

Optimal control run III

However, a smaller $\alpha_{1}>0$ will give a smaller $\mathrm{TKE}_{\mathrm{final}}$, while maintaining temporal smoothness in the controls.

TABLE 1.

\begin{tabular}{c|ccc|ccc} 
Run & $\alpha_{1}$ & $\alpha_{2}$ & $\alpha_{3}$ & $J_{0}$ & $J_{\text {final }}$ & $\mathrm{TKE}_{\text {final }}$ \\
\hline I & 0.5 & 0.005 & 0.005 & 12.43 & 0.48 & 0.42 \\
II & 0.05 & 0.005 & 0.005 & 12.43 & 0.37 & 0.32 \\
III & 0 & 0.005 & 0.005 & 12.43 & 0.24 & 0.20
\end{tabular}

In all cases the optimization is started with zero control. The optimal wall-normal velocity distributions $g_{2}$ for are plotted in Figure 1. The optimal controls for runs I and II are very similar, the amplitudes in the optimal control for runs II are slightly higher than those for run I, but no additional oscillations arise when $\alpha_{1}$ is reduced to 0.05 . The plots clearly show the effect of the regularization term $\int \frac{\alpha_{1}}{2}\left\|\mathbf{g}_{t}\right\|$. Without it, the control starts to oscillate in time in the second half of $\left[t_{0}, t_{f}\right]$ and it exhibits a large jump at $t_{0}$. If we even set $\alpha_{1}=0$ and $\alpha_{2}=0$, then controls are produced that exhibit strong spatial and temporal oscillations which frequently led to a failure in the compressible Navier-Stokes solver.

Figure 2 shows the contours of kinetic energy for the uncontrolled flow and the controlled flow, run I.

\section{Acknowledgements}

The work of S. Collis, K. Ghayour and M. Heinkenschloss was supported by Texas ATP grant 003604-0001, 1999. M. Ulbrich and S. Ulbrich were supported by DFG grants 
FIGURE 2. Kinetic energy contours for the uncontrolled flow (top row) and the controlled flow, run I (bottom row).
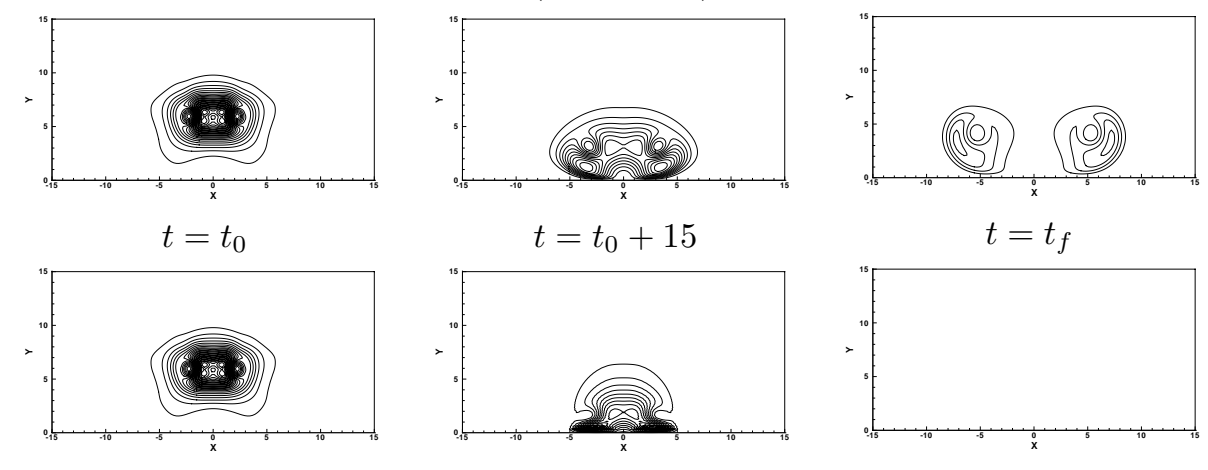

$t=t_{0}$

$t=t_{0}+15$

$t=t_{f}$

UL157/3-1 and UL158/2-1, respectively, and by CRPC grant CCR-9120008. Computations were performed on an SGI Origin 2000 which was purchased with the aid of NSF SCREMS grant 98-72009.

\section{References}

[1] M. Berggren, Numerical solution of a flow-control problem: Vorticity reduction by dynamic boundary action, SIAM J. Scientific Computing, 19 (1998), 829-860.

[2] T. R. Bewley, P. Moin, and R. Teman, DNS-based predictive control of turbulence: an optimal target for feedback algorithms, Submitted to J. Fluid Mech., 2000.

[3] E. M. Cliff, M. Heinkenschloss, and A. Shenoy, Airfoil design by an all-at-once method, International Journal for Computational Fluid Mechanics, 11 (1998), 3-25.

[4] S. S. Collis, K. Ghayour, M. Heinkenschloss, M. Ulbrich, and S. Ulbrich, Towards adjointbased methods for aeroacoustic control, in: 39th Aerospace Science Meeting \& Exhibit, January 8-11, 2001, Reno, Nevada, AIAA Paper 2001-0821 (2001).

[5] S. S. Collis, Y. Chang, S. Kellogg, and R. D. Prabhu, Large Eddy Simulation and Turbulence Control, AIAA paper 2000-2564, (2000).

[6] T. Colonius, S. K. Lele, and P. Moin, The Free Compressible Viscous Vortex, J. Fluid Mech. 230 (1991), pp. 45-73.

[7] M. Gad el Hak, A. Pollard, and J.-P. Bonnet, editors, Flow Control. Fundamental and Practices, Springer Verlag, Berlin, Heidelberg, New York, 1998.

[8] A. V. Fursikov, Optimal Control of Distributed Systems. Theory and Applications, Translation of Mathematical Monographs 87, American Mathematical Society, Providence, Rhode Island, 2000.

[9] A. V. Fursikov, M. D. Gunzburger, and L. S. Hou, Boundary value problems and optimal boundary control for the Navier-Stokes systems: The two-dimensional case, SIAM J. Control Optimization 36 (1998), 852-894. 
[10] M. D. Gunzburger and L. S. Hou, editors, International Journal of Computational Fluid Dynamics, No. 1-2, 11 (1998).

[11] M. D. Gunzburger, L. S. Hou, and T. P. Svobotny, Optimal control and optimization of viscous, incompressible flows, in: M. D. Gunzburger and R. A. Nicolaides, editors, Incompressible Computational Fluid Dynamics, Cambridge University Press, New York, (1993) 109-150.

[12] M. D. Gunzburger and S. Manservisi, The velocity tracking problem for Navier-Stokes flows with boundary control, SIAM J. Control Optim., 39 (2000), 594-634.

[13] D. Hoff, Discontinuous solutions of the Navier-Stokes equations for multidimensional flows of heat-conducting fluids, Arch. Rational Mech. Anal., 139 (1997), 303-354.

[14] A. Jameson, L. Martinelli, and N. A. Pierce, Optimum aerodynamic design using the NavierStokes equations, Theor. and Comput. Fluid Dynamics, 10 (1998), 213-237.

[15] A. Jameson and J. Reuther, Control theory based airfoil design using the Euler equations, AIAA Paper 94-4272, (1994).

[16] W. H. Jou, W. P. Huffman, D. P. Young, R. G. Melvin, M. B. Bieterman, C. L. Hilems, and F. T. Johnson, Practical considerations in aerodynamic design optimization, in: Proceedings of the 12th AIAA Computational Fluid Dynamics Conference, San Diego, CA, June 19-22 1995. AIAA Paper 95-1730.

[17] P. L. Lions, Mathematical Topics in Fluid Mechanics. Volume 2, Compressible Models. Oxford Lecture Series in Mathematics and its Applications 10, Claredon Press, Oxford, 1998.

[18] A. Matsumura and T. Nishida, The initial value problem for the equations of motion of viscous and heat-conductive gases, J. Math. Kyoto Univ., 20 (1980), 67-104.

[19] A. Matsumura and T. Nishida, Initial-boundary value problems for the equations of motion of compressible viscous and heat-conductive fluids, Comm. Math. Phys. 89 (1983), 445-464.

[20] J. Nocedal and S. J. Wright, Numerical Optimization, Springer Verlag, Berlin, Heidelberg, New York, 1999.

[21] B. Soemarwoto, The variational method for aerodynamic optimization using the NavierStokes equations, Technical Report 97-71, ICASE, NASA Langley Research Center, Hampton VA 23681-0001, 1997.

[22] A. Valli, Mathematical results for compressible flows, in: J. F. Rodriguez and A. Sequeira, editors, Mathematical Topics in Fluid Mechanics, Pitman Research Notes Mathematics 274, Longman Scientific and Technical, Essex, (1992) 193-229.

Departments of Computational and Applied Mathematics and of Mechanical Engineering and Materials Science,

Rice University,

Houston, TX 77005-1892, USA

E-mail address: collis@rice.edu, kghayourarice.edu, heinken@rice.edu

Lehrstuhl für Angewandte Mathematik und Mathematische Statistik,

Technische Universität München,

D-80290 München, Germany

E-mail address: mulbrich@ma.tum.de, sulbrich@ma.tum.de 